\title{
Reducing handoff delay by adaptive threshold tuning and location updates in mobile WiMAX
}

\author{
Dr.B.Sridevi ${ }^{1}$, Akshatha chindu. $\mathrm{M}^{2}$, G.Abinaya ${ }^{3}$ \\ ${ }^{1}$ Assistant Professor, ${ }^{2,3 U . G}$ Student \\ Department of Electronics and Communication Engineering, Velammal College of \\ Engineering and Technology,
}

\begin{abstract}
In wireless networks data are sent and received with impressive speed and ease. Mobile WiMAX is a broadband wireless solution that enables coverage of mobile and fixed broadband networks with flexible network architecture. Devised as a truly broadband access solution, the WiMAX technology offers promising features in terms of high bandwidth, extended coverage area and low cost. Despite having many advantages, WiMAX faces major research issues like QoS based bandwidth allocation, Roaming, Internetworking with other technologies, security and handoff. Handoff occurs when a mobile user goes from one cell to another without interruption of ongoing session. Many approaches have been proposed for reducing handoff delay. In this paper, we propose a new approach by which a critical area, an area far away from serving base station but still inside the coverage of serving base station, is found. And then overlapping area between serving base stations and neighboring base station are found and tabulated. According to the size of overlapping area, different weights are assigned where handoff threshold is changed adaptively to reduce handoff delay.
\end{abstract}

\section{KEYWORDS}

Hard handoff, handoff delay, handoff threshold

\section{INTRODUCTION}

In the last few years, WiMAX (worldwide interoperability for microwave access) has been proposed as a promising wireless communication technology since it provides high data rate communications in metropolitan area networks (MANs). According to the standards, WiMAX can support up to a $75 \mathrm{Mbps}$ data rate and cover up to 30 miles. Mobile WiMAX was the first mobile broadband wireless-access solution based on the IEEE 802.16e-2005 standard that enabled convergence of mobile and fixed broadband networks through a common wide-area radio-access technology and flexible network architecture. For a Mobile station, mobility is one of the truly distinctive standards that wireless offers. WiMAX is one of the crucial challenges that is faced by Mobile WiMAX. Handoff mechanism handles a mobile station switching from serving base station (SBS) to target base station (TBS). in general handoff are of two types, hard handoffs and soft handoff. Hard Handoff is the default handover mechanism, whereas soft handover Mechanism is the optional procedure. Hard handover is also known as break-beforemake. The subscriber mobile station (MS) stops its radio link with the first Base Station before establishing its radio link with the new Base Station. This is a rather simple handover. The two soft handoffs mechanisms are Macro-Diversity Handoff (MDHO) and Fast Base Station Switching (FBSS).Soft handover, also known as make-before-break. The Mobile Station

DOI : $10.5121 /$ ijist.2014.4316 
establishes its radio link with a new Base Station before stopping its radio link with the first Base Station. The Mobile Station may have two or more links with two or more Base Stations, which gives the soft handover state. The soft handover is evidently faster than the hard handover.

The HHO scheme in 802.16e is highly bandwidth-efficient and seamless in nature. HHO is the simplest Mobile WiMAX technique that ensures efficient support for the provisioning of different high-speed real time applications without significant interruptions and QoS degradation. The major advantages of the HHO scheme in Mobile WiMAX are the low deployment complexity and cost, requiring very few BSs spaced appropriately apart. The entire process of Hard Handoff procedure in Mobile WiMAX is broadly categorized into two phases namely Network Topology Acquisition Phase and the Actual Handoff Phase.

Network Topology Acquisition Phase is a cell reselection stage in which a suitable neighboring base station (NBS) is selected for handoff. There are three steps in this phase. First step is BS advertisement, in which the SBS periodically broadcasts information about different NBSs for handoffs by a MAC-management message called Mobile Neighbor Advertisement (MOB NBR_ADV).Second step is Scanning and synchronization of BSs by MS, where in order to start the scanning procedure, the MS sends a scanning interval allocation request (MOB_SCN-REQ) to the SBS containing a list of potential NBSs, selected from the MOB_NBR-ADV broadcasts. In response, the SBS sends back a scanning response (MOB_SCN-RSP) message to the MS allocating scanning intervals for the scanning procedure. The response message also contains information about the specified starting frame of the scan procedure, the length of interleaving intervals as well as the number of scan iterations. The MS thus scans the selected NBSs within allotted time frames to select suitable candidate BSs for the handoff. Scanning results in selection of a list of potential candidate BSs for handoff with the help of a scanning result report (MOB_SCN-REP) message. Third Step is Ranging and Other Activities, in which MS collects some information regarding TBSs using MAC management messages like Ranging Request (RNG-REQ) and Ranging Response (RNG-RSP) that are exchanged between MS and NBS for. Until the fine tuning between the MS and the respective BSs are completed, repeated ranging request and response steps take place. In actual handoff phase a particular TBS from the list of candidate NBSs selected during the Network Topology Acquisition Phase is chosen for the handoff process. Firstly selection of target base station takes place, in which the MS communicates a handoff request message (MOB_MSHO-REQ) to the SBS with the identity and measurement report of selected NBSs. On receiving the message, the SBS negotiates with the potential TBSs regarding QoS and other important resources to support the MS after the handoff activity. From the received replies, the SBS summarizes and communicates a short list of recommended NBSs to the MS through the handoff response (MOB_BSHO-RSP) message, the MS quickly decides and sends a prompt handoff indication (MOB_HO-IND) message to the SBS with the details of the finalized TBS. Then the connection between MS and SBS is disconnected. The next step is MS Synchronization with the Selected TBS, where the MS performs time and frequency synchronization with the TBS. In the Ranging and Network ReEntry step in which MS and the TBS exchange their supported parameters through the communication of SS basic capability request (SBC-REQ) and response (SBC-RSP) messages which in turn is followed by MS Authorization, Registration of the MS. IP connectivity is established. Last step is Termination of MS Contexts where, the previous SBS terminates all kinds of MS-related connections and contexts associated with them.

\section{RELATED WORKS}

Handoff process is a predominant issue in mobile WiMAX. Several papers were published 
addressing Handoff related issues. In [1], A survey of MAC, cross-layer and network scenario issues was discussed with various solutions along with comparative study. To improve the performance of the existing IEEE 802.16e network, IEEE802.16j has been developed which provides multihop relay technologies. In [2] a relay station grouping performance has been investigated and an algorithm has been developed to minimize handoffs by using greedy grouping policy. To provide secure and seamless services for mobile users [3] proposes fast and secure inter ASN handoff to overcome the denial of service and replay attacks using less communication and computation resources. In [4] a group based handoff authentication scheme which not only satisfies necessary security requirements but also achieves privacy presentation is proposed. [5] Proposes hierarchical design concept of a preamble for orthogonal frequency division multiplexing (OFDM) based mobile mesh networks to reduce handoff overhead. In [6] a multiple trigger initiated handoff execution is proposed which considers signal strength along with hysteresis and threshold, free capacity estimation and dependence on mobile velocity during handoff process. The two fast handoff mechanisms, fast base station switching and macro diversity handoff, operate with diversity set containing base station list. In [7] a predictive handoff approach is developed using numerical extrapolation method that accommodates temporal locality of mobile station. Usually the scanning along with association scheme introduces additional handoff latency. In [8] conventional scanning is evaluated and a mechanism to reduce association latency is proposed. [9] Proposed a serving base station controlled fast target base station (TBS) selection scheme which utilized the principle of orientation matching between geographical position of each NBS and MS direction of motion and signal strengths of different NBSs. The SBS selects the TBS out of shortlisted NBSs. Handoff latency and packet loss ratio affects the quality of mobile wireless networks. In [10] a fast handoff scheme using mobility patterns for WiMAX networks is proposed. In [11] a mobility prediction method based on fuzzy logic control to predict the movement patterns and handoff times by measuring mobile station's velocity and received signal strength of SBS and TBS simultaneously is proposed. Velocity is an important factor in handoff initiation process. [12] Proposed a scheme that adopts dynamic handoff threshold according to velocity that reduces handoff delay and improves network resource -utilization. In [13] an improved mobility handoff algorithm was proposed that skips some unnecessary handoff stages, reduces handoff delay by adjusting certain handoff parameters. A pre-authentication [14] scheme is proposed which skips EAP authentication if mobile station moves from one base station to another in the same network, thus reducing handoff latency.

\section{PROPOSED WORK}

In our proposed work, critical area and overlapping coverage area between neighboring base station and current serving base station (SBS) are predicted. In order to serve the above process handoff threshold is tuned adaptively thus reducing handoff delay and number of scans to determine the target base station

Critical area (CA) is an area that is far away from the serving base station but still inside the coverage range of current serving base station. When a mobile station reaches this critical area, handoff process will be initiated by sending MOB_NBR_ADV to the MS. figure 1 shows the critical area. Overlapping area is an area between a base station and its neighbor base stations area. The area corresponds to overlapping received signal strengths of the two base stations, as shown in figure 2.

Each base station contains a table of neighboring base stations with their respective overlapping areas. Weights are assigned according to the overlapping area. If the overlapping area is large, 
high weight is assigned to it. If the overlapping area is small, low weight is assigned to it. Whenever a weight is large, there is more probability of MS to move towards that particular neighboring base station. Each SBS has a mobility pattern table, as shown in table1, containing previous base station ID, target base station ID and handoff times. A high handoff priority is given to a NBS that has large weight and high handoff times in mobility pattern table. A low handoff priority is assigned to a NBS that has small weight and low handoff times in mobility pattern table, thus allowing the SBS itself to serve the MS. Handoff threshold is tuned adaptively according to the given handoff priority. If any two weights are equal then normal handoff process takes place

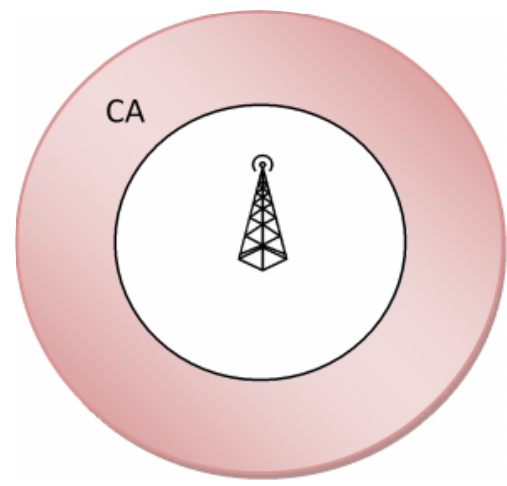

Figure 1: critical area

\begin{tabular}{|c|c|c|}
\hline Previous BS & Target BS & Handoff times \\
\hline BS1 & BS3 & 104 \\
\hline BS6 & BS7 & 95 \\
\hline BS1 & BS5 & 59 \\
\hline$\ldots$ & $\ldots$ & $\ldots$ \\
\hline
\end{tabular}

Table 1 : A sample mobility pattern table

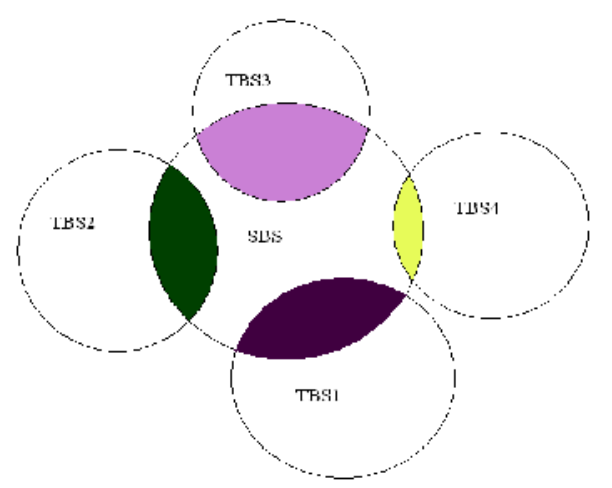

Figure 2: overlapping areas between SBS and many TBS 


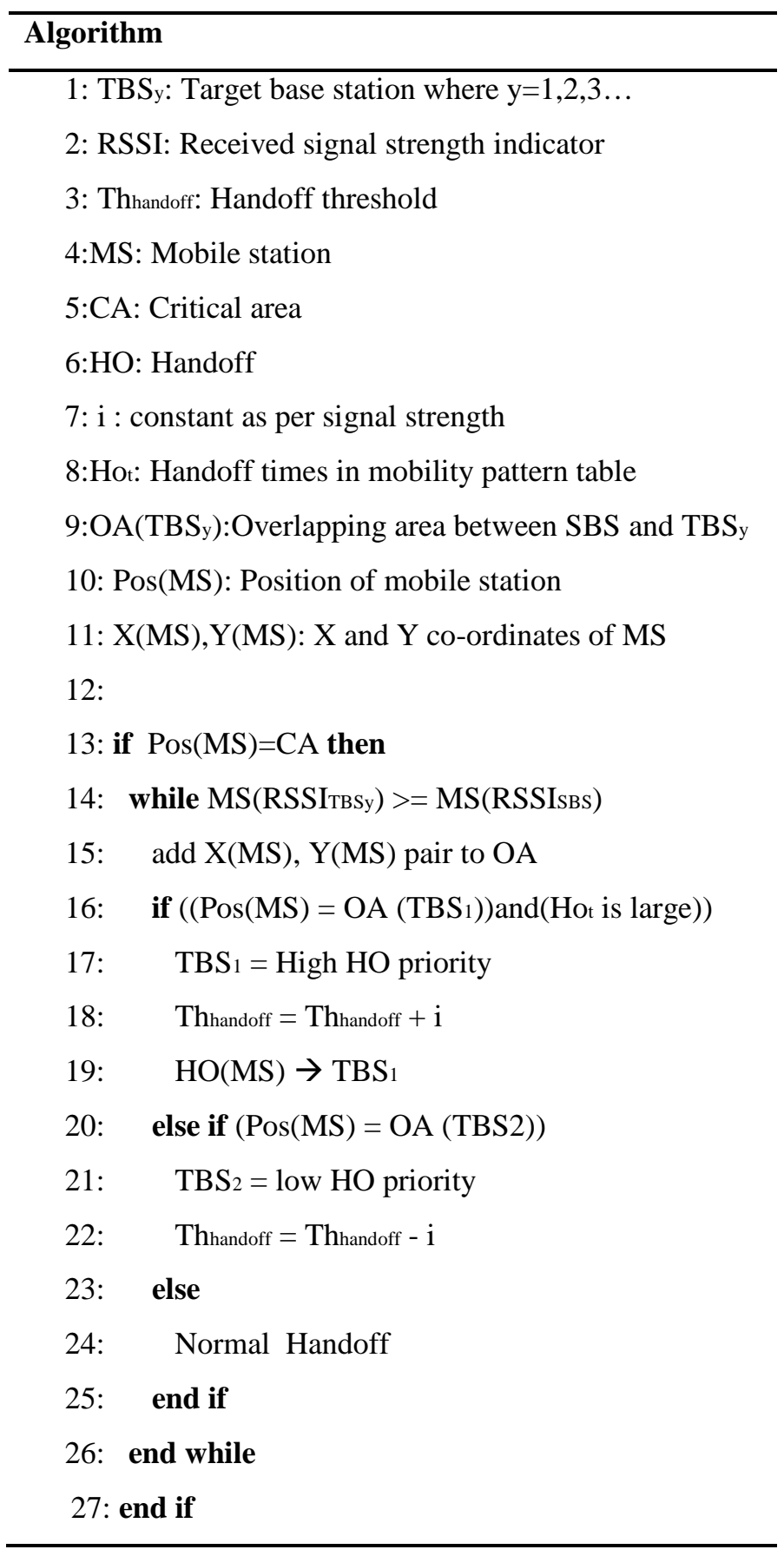

\section{RESULTS AND DISCUSSION}

We created a simple WiMAX scenario in OPNET modeler 14.5.Trajectories were given to certain mobile stations, as shown in figure 3.After implementing our algorithm and running discrete simulation event, we observed the serving base station id and handoff delay of certain mobile stations and compared the results with the handoff process in existing model 
Figure 4(a) and (b) shows the Serving base station id of the mobile_1_1 and the delay associated with it during the handoff process. Figure 4(a) shows the normal WiMAX scenario where two handoffs take place, from BS1 to BS4 and from BS4 to BS5. And figure (b) shows the proposed work result where only one handoff takes place from BS1 to BS5. We inferred that handoff delay is reduced; unnecessary handoffs are avoided, which in turn reduces resource wastage.

Figure 5(a) shows the normal WiMAX scenario of mobile_1_4, where two handoffs take place, from BS1 to BS4 and from BS4 to BS3. And figure 5(b) shows the proposed work result where only one handoff takes place from BS1 to BS3, thus reducing handoff delay. Figure 6(a) and 6(b) shows the similar results of mobile_2_1 in base station 2 and results 7(a) and 7(b) corresponds to results of mobile_6_4 served by base station 6

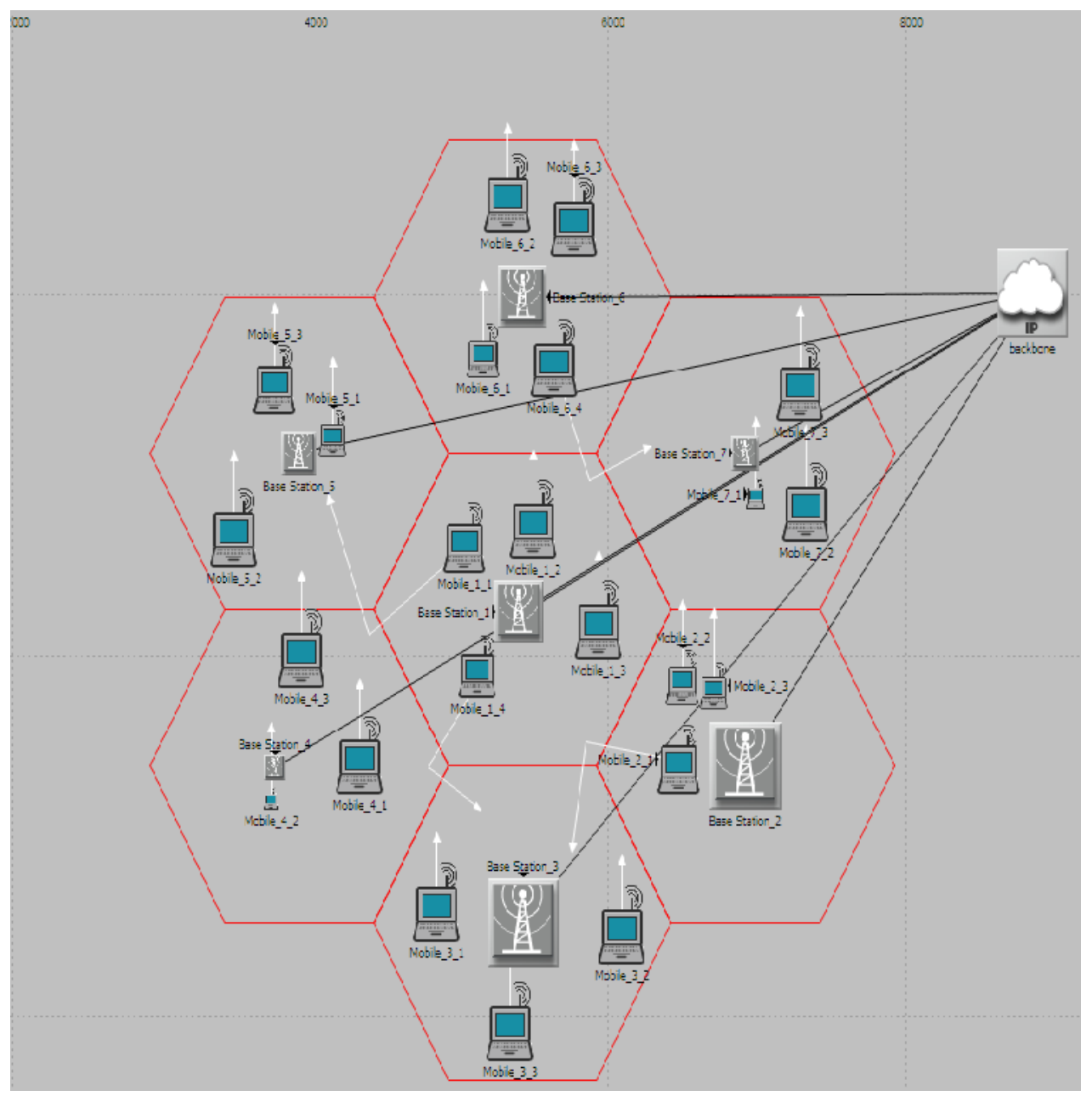

Figure 3. WiMAX scenario 
International Journal of Information Sciences and Techniques (IJIST) Vol.4, No.3, May 2014

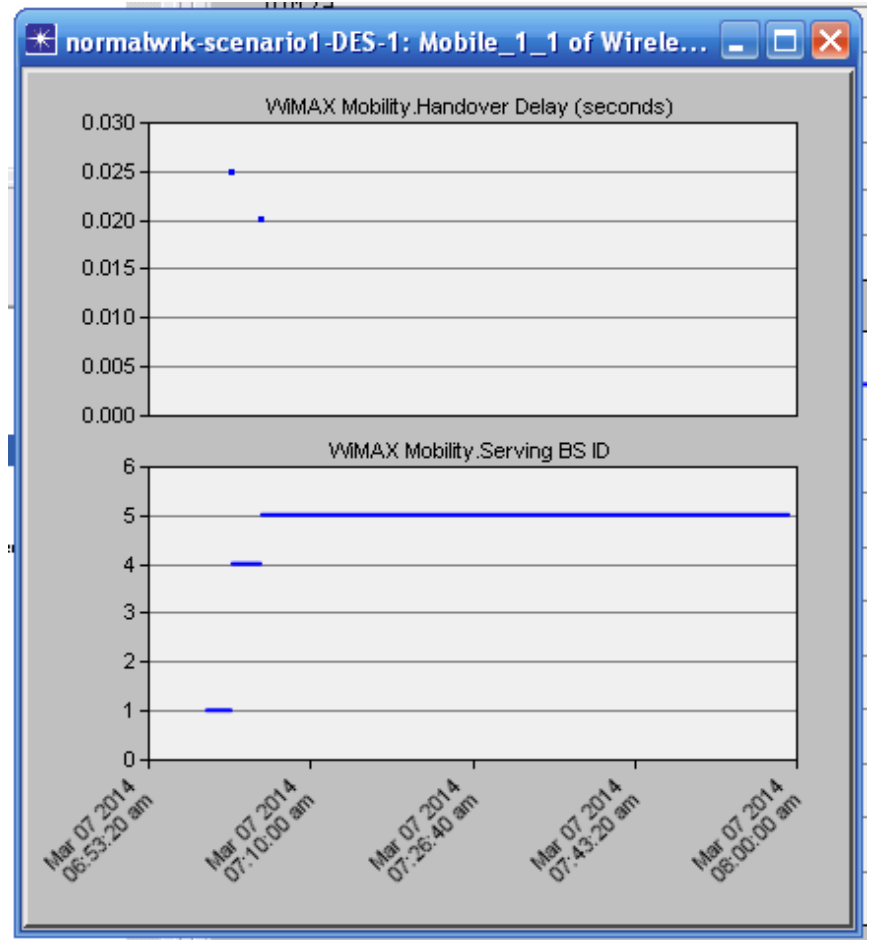

Figure 4 (a) Mobile 1_1 in base station 1 (existing work)

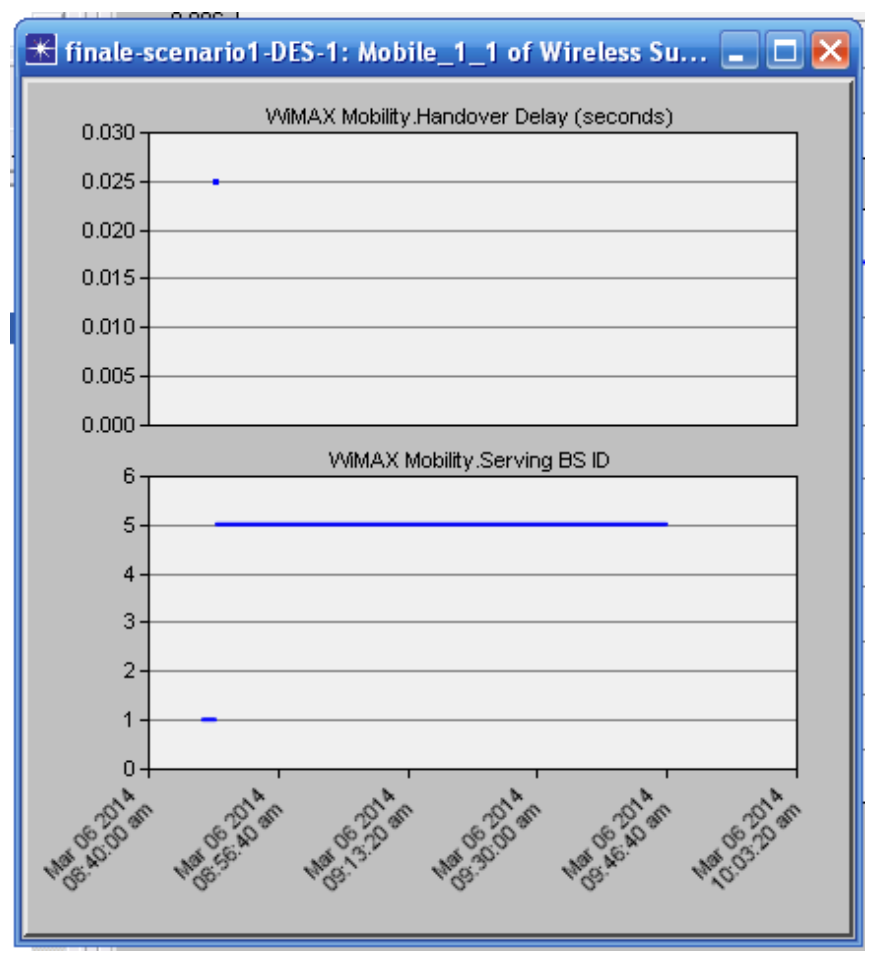

Figure 4 (b) Mobile 1_1 in base station1 (proposed work) 
International Journal of Information Sciences and Techniques (IJIST) Vol.4, No.3, May 2014

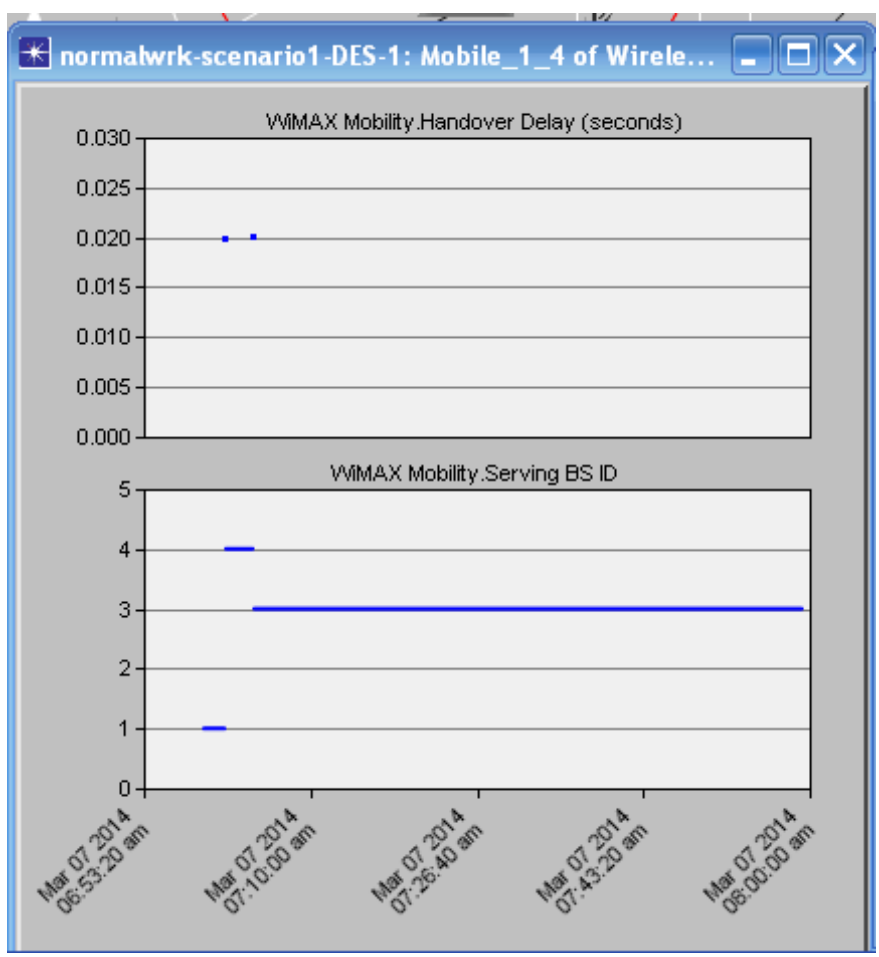

Figure 5 (a) Mobile 1_4 in base station 1 (existing work)

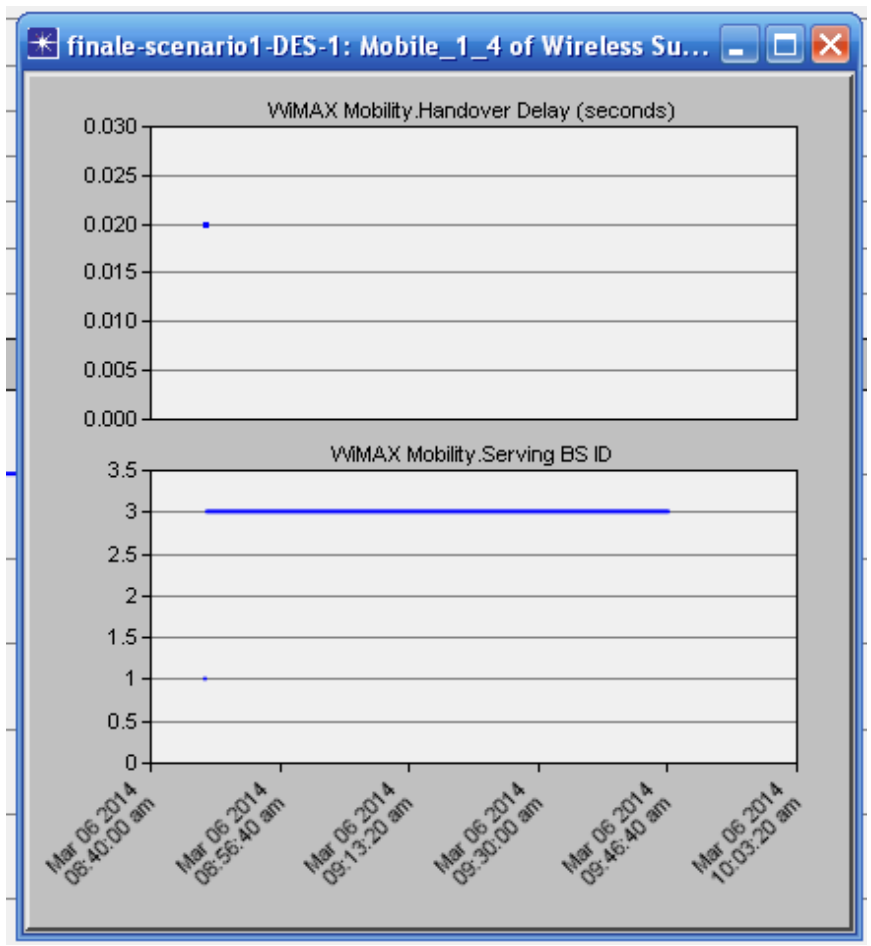

Figure 5 (b) Mobile 1_4 in base station1 (proposed work) 
International Journal of Information Sciences and Techniques (IJIST) Vol.4, No.3, May 2014

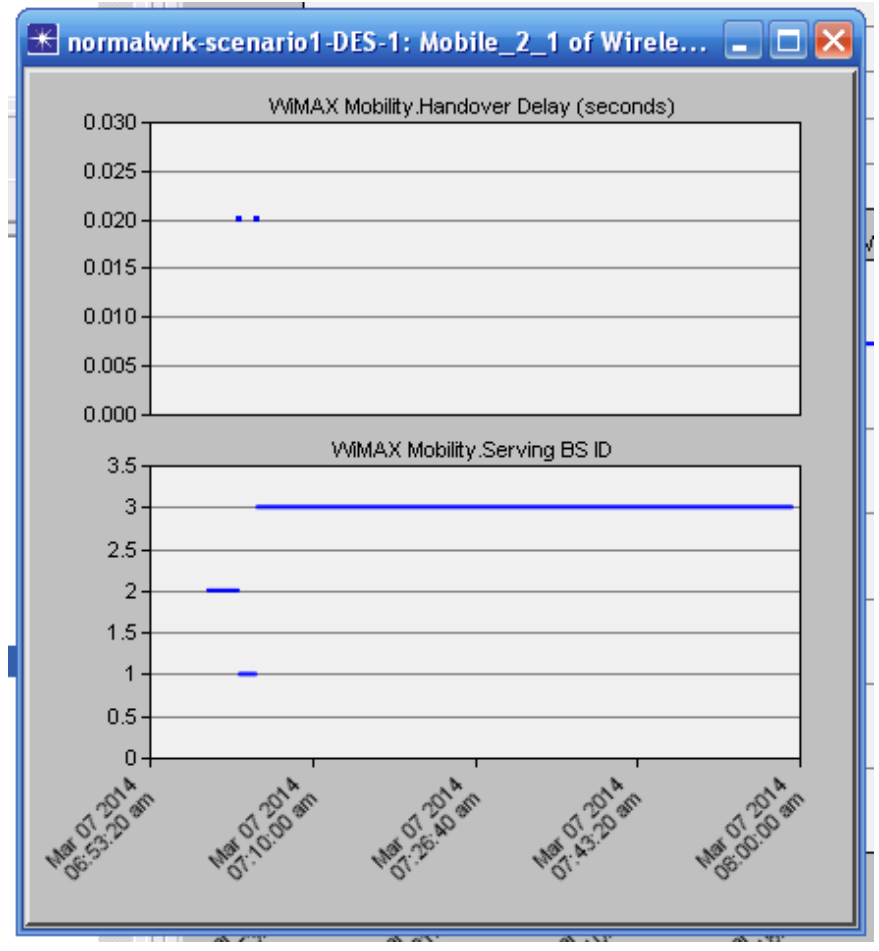

Figure 6 (a) Mobile 2_1 in base station 2 (existing work)

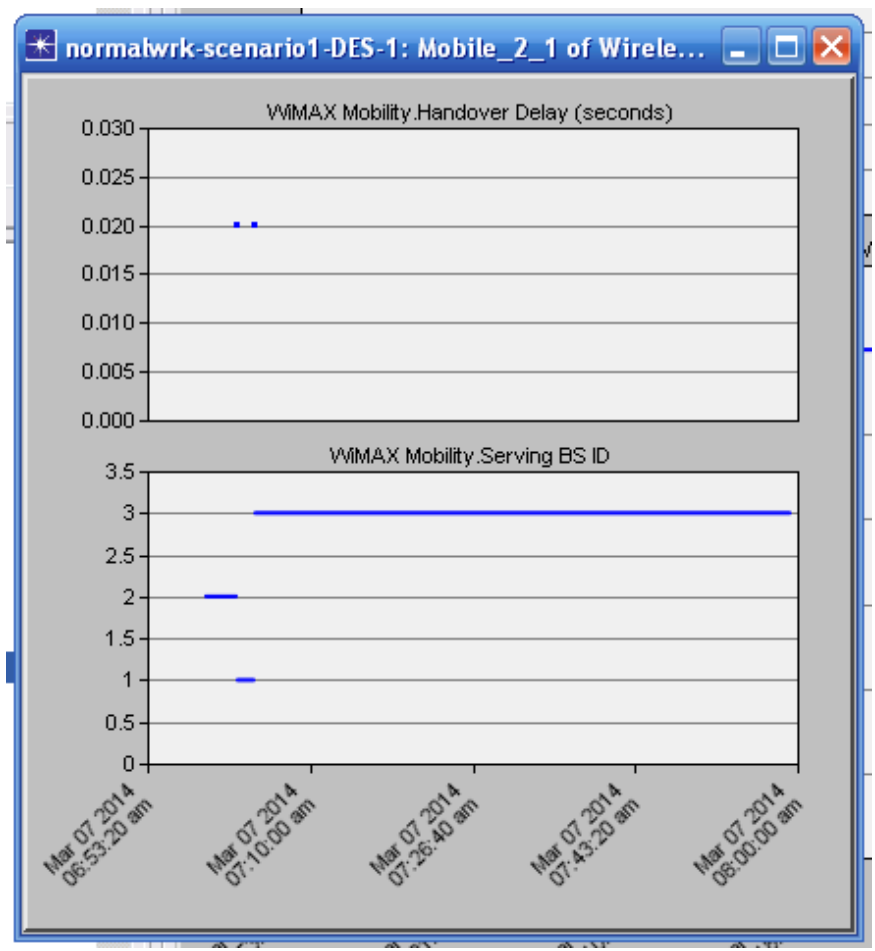

Figure 6 (a) Mobile 2_1 in base station 2(proposed work) 
International Journal of Information Sciences and Techniques (IJIST) Vol.4, No.3, May 2014

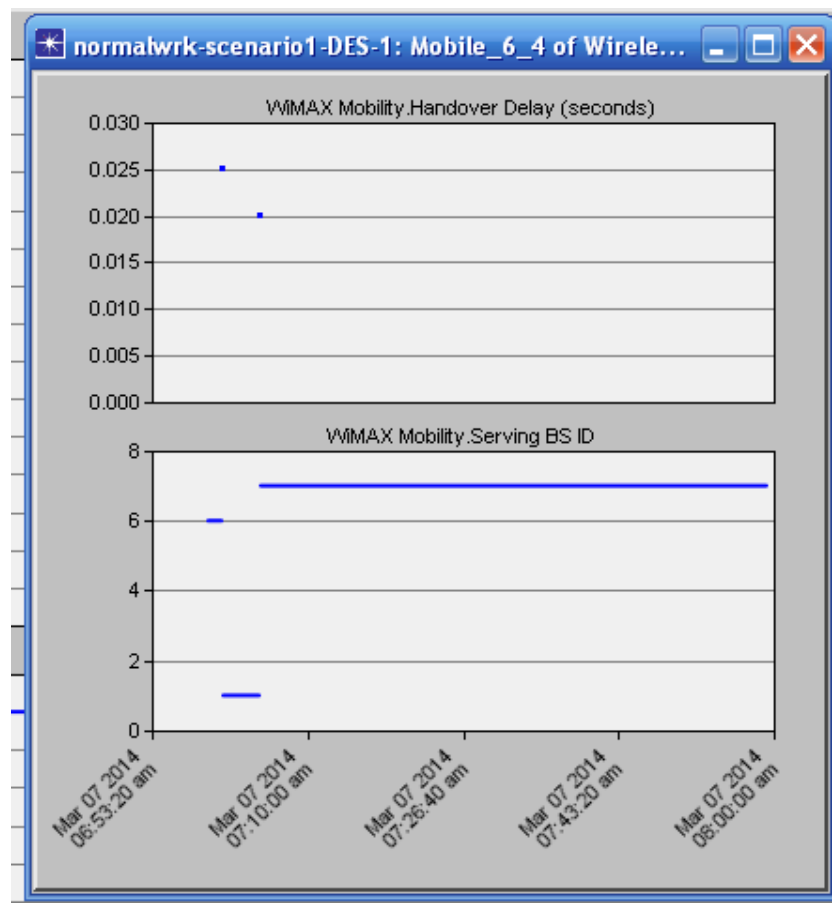

Figure 7 (a) Mobile 6_4 in base station 4 (existing work)

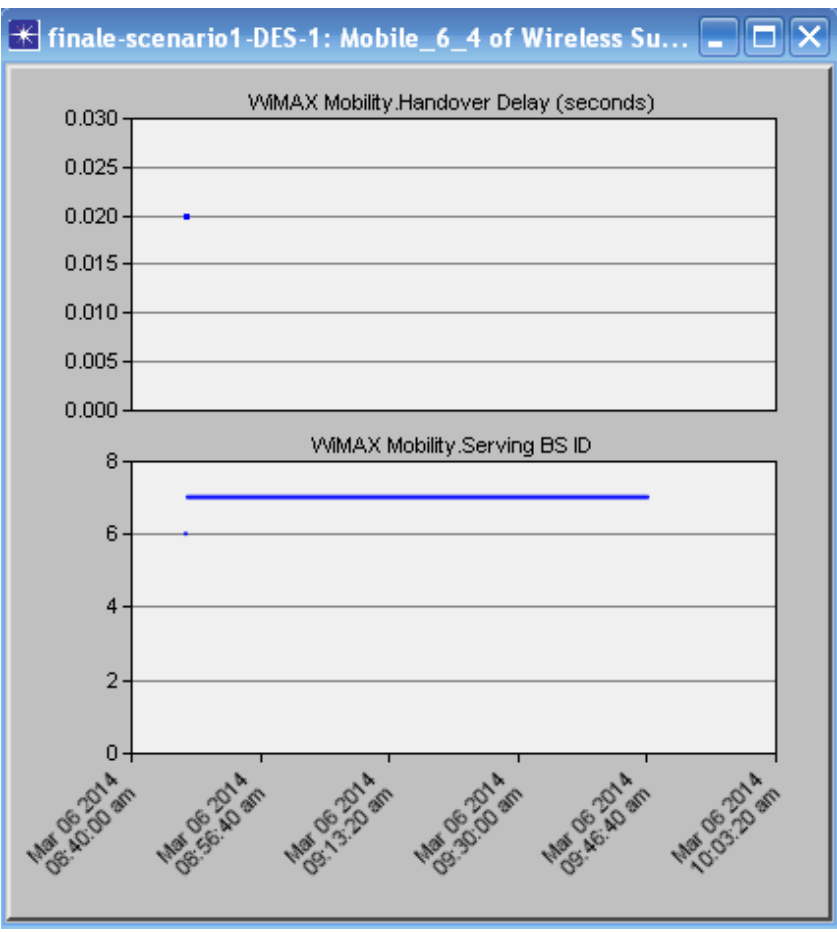

Figure 7(b) Mobile 6_4 in base station 4(proposed work) 


\section{Conclusion}

In this paper, to minimize handoff delay, avoid unnecessary handoffs and reduce number of target base stations, we propose a new protocol based on overlapping area and mobility pattern table. Our simulation results showed proposed protocol can roughly achieve 50 percent reduction in handoff delay, skipping unnecessary handoff are reduced. In future work, we have in mind to analyze the security related issues and also QoS management of the proposed protocol. A comparative study with existing protocols and improvements beside WiMAX standard will be made.

\section{REFERENCES}

[1] Sayan Kumar Ray ,Krzysztof Pawlikowski, Harsha Sirisena, "handover in mobile wimax networks: the state of art and research issues", IEEE Communications Surveys and Tutorials Magazine, 2010

[2] Shun-Ren Yang, Chien-Chi Kao, Wai-Chi Kan, Tzung-Chin Shih,"Handoff Minimization through a Relay Station Grouping Algorithm with Efficient Radio-Resource Scheduling Policies for IEEE 802.16j Multihop Relay Networks", IEEE transactions on vehicular technology, june 2010

[3] Thuy Ngoc Nguyen, Maode Ma," Enhanced EAP-Based Pre-Authentication for Fast and Secure Inter-ASN Handovers in Mobile WiMAX Networks", IEEE transactions on wireless communications, June 2012.

[4] Anmin Fu, Shaohua Lan, Bo Huang, Zhenchao Zhu, and Yuqing Zhang," A Novel Group-Based Handover Authentication Scheme with Privacy Preservation for Mobile WiMAX Networks", IEEE communications letters, november 2012

[5] Hyunil Yoo, Mina Lee, Tae Howan Hong, and Yong Soo Cho.” A Preamble Design Technique for Efficient Handover in IEEE 802.16 OFDM-Based Mobile Mesh Networks", IEEE transactions on vehicular technology, January 2013

[6] P.P. Edwin Winston, K.S Shaji,” Multiple Trigger Initiated Handover Execution In WiMAX Networks", International Conference on Circuits, Power and Computing Technologies, 2013

[7 ]Kuang-Hui Chi, J. Morris Chang, Ting-Chung Wang,” Enhanced Fast Base Station Switching”, IEEE transactions on mobile computing, November 2012

[8] You-Lin Chen,Shiao-Li Tsao,"A Low-Latency Scanning with Association Mechanism for RealTime Communication in Mobile WiMAX”, IEEE transactions on wireless communications, october 2012

[9] Sayan Kumar Ray, Harsha Sirisena, Devatanu Deka," Fast and Reliable Target Base Station Selection Scheme for Mobile WiMAX Handover",IEEE, 2012

[10] Zhenxia Zhang, Richard W. Pazzi , Azzedine Boukerche, Bjorn Landfeldt," Reducing Handoff Latency for WiMAX Networks using Mobility Patterns",IEEE WCNC ,2010

[11] Mohammed K. Al-Khateeb1, Aisha-Hassan A. Hashim2, "A Fuzzy-based Mobility Prediction in the IEEE 802.16e", International Journal of Communication Networks and Information Security (IJCNIS), April 2009

[12] Caiyong HAO, Hongli LIU, Jie ZHAN," A Velocity-Adaptive Handover Scheme For Mobile WiMAX", Int. J. Communications, Network and System Sciences, 2009

[13] Imtiyaz Anwar, Arun Khosla "A Mobility Improvement Handover Scheme for Mobile-WiMAX", International Journal of Computer Applications, vol. II, no. 3, December 2010. 
International Journal of Information Sciences and Techniques (IJIST) Vol.4, No.3, May 2014

\section{Authors}

Dr.B.Sridevi, Professor of ECE Department of Velammal College of Engineering \& Technology, Madurai, obtained her B.E., degree from A.C.C.E.T Karaikudi , Madurai Kamaraj University, Madurai and M.E. degree from Anna University, Chennai. She has 2 years of Industrial experience, 10 years of Teaching, and Research experience. Completed Ph.D. in Anna University, Chennai in WiMAX Networks. She have published many research papers in International journals, national and international

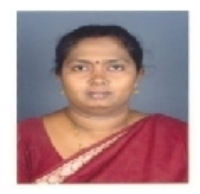
conferences. Her area of research includes Network Security, Wireless Networks. Email id: aisveriya@yahoo.com

Akshatha chindu.M , Under Graduate scholar, pursuing B.E degree in the Department of Electronics and Communication Engineering at Velammal College of Engineering and Technology, Madurai. Her area of interest includes Computer Networks, Network Security, and Wireless Networks. Email id: akshathachindu1992@gmail.com

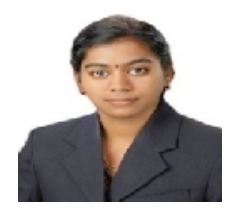

G.Abinaya , Under Graduate scholar, pursuing B.E degree in the Department of Electronics and Communication Engineering at Velammal College of Engineering and Technology, Madurai. Her area of interest includes Computer Networks, Network Security, and Wireless Networks. Email id: abinayagiri1992@gmail.com

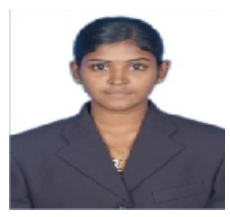


International Journal of Information Sciences and Techniques (IJIST) Vol.4, No.3, May 2014 